\title{
Long-term outcomes and prognosis for patients with sarcomatoid hepatocellular carcinoma
}

\author{
Cheng Zhou, ${ }^{1,2 \#}$, Xin Zhang ${ }^{3 \#}$, Kaiqian Zhou ${ }^{1,2 \#}$, Yingyong Hou ${ }^{3}$, Feiyu Chen ${ }^{1,2}$, Xiangyu Zhang ${ }^{1,2}$, Yuan $\mathrm{Ji}^{3}$, \\ Shuangjian Qiu, ${ }^{1,2}$, Jia Fan ${ }^{1,2,5}$, Jian Zhou ${ }^{1,2,4,5}$, Yuhong Zhou ${ }^{6}$, Zheng Wang ${ }^{1,2}$ \\ ${ }^{1}$ Liver Cancer Institute, Zhongshan Hospital, Fudan University, Shanghai, China; ${ }^{2}$ Key Laboratory of Carcinogenesis and Cancer Invasion, Ministry \\ of Education, Fudan University, Shanghai, China; ${ }^{3}$ Department of Pathology, Zhongshan Hospital, Fudan University, Shanghai, China; ${ }^{4}$ Institute of \\ Biomedical Sciences, Fudan University, Shanghai, China; ${ }^{5}$ State Key Laboratory of Genetic Engineering and Collaborative Innovation Center for \\ Genetics and Development, School of Life Sciences, Fudan University, Shanghai, China; ${ }^{6}$ Department of Medical Oncology, Zhongshan Hospital, \\ Fudan University, Shanghai, China \\ Contributions: (I) Conception and design: Z Wang, K Zhou; (II) Administrative support: Y Hou, F Chen, X Zhang; (III) Provision of study \\ materials or patients: Y Ji, S Qiu, J Fan, J Zhou, Y Zhou; (IV) Collection and assembly of data: C Zhou, X Zhang, K Zhou; (V) Data analysis and \\ interpretation: C Zhou, X Zhang; (VI) Manuscript writing: All authors; (VII) Final approval of manuscript: All authors. \\ "These authors contributed equally to this work. \\ Correspondence to: Zheng Wang, MD, PhD. Professor of Medicine, Liver Cancer Institute, Zhongshan Hospital, Fudan University, Shanghai 200030, \\ China. Email: wzdoc@163.com; wang.zheng@zs-hospital.sh.cn.
}

Background: Characterized by spindle cell composition in hepatocellular carcinoma tumor, sarcomatoid hepatocellular carcinoma (SHC) is a rare malignant with poor prognosis. In this study, we aimed to evaluate the clinical and pathological features of SHC and establish a nomogram that can predict long-term outcomes of the disease.

Methods: We retrospectively analyzed 63 patients who were diagnosed with SHC between October 2007 and November 2016 and used immunohistochemistry (IHC) to assessed various markers in liver samples. The clinical data and the histological and pathological findings were collected and used to build a nomogram to predict survival.

Results: The median overall survival (OS) and the recurrence-free survival (RFS) in SHC were 23.2 and 8.4 months, respectively. High expression levels of tyrosine-protein kinase Met (17/63, 27.0\%) were associated with poorer RFS $(\mathrm{P}=0.040)$. A panel of markers, consisting heat-shock protein 70 (HSP70), glutamine synthetase (GS), and glypican-3 (GPC3), merged as an independent risk factor for treatment outcomes. The nomogram, which including this panel of markers, predicted OS times with a concordanceindex (C-index) score of 0.758 (95\% CI: 0.672-0.843) in the training set and 0.832 (95\% CI: 0.712-0.952) in the validation set. The use of the nomogram showed marked improvements in the prediction of patient outcomes compared with conventional staging systems $(\mathrm{P}<0.05)$.

Conclusions: Diagnosis of SHC is rare and has a relatively poor prognosis. A panel of markers HSP70, GS and GPC3 served as an independent prognostic factor for SHC.

Keywords: Sarcomatoid hepatocellular carcinoma (SHC); heat-shock protein 70 (HSP70); glutamine synthetase (GS); glypican-3 (GPC3); nomogram

Submitted Aug 17, 2021. Accepted for publication Dec 31, 2021.

doi: $10.21037 /$ atm-21-4322

View this article at: https://dx.doi.org/10.21037/atm-21-4322 


\section{Introduction}

Sarcomatoid carcinoma is an extremely rare tumor composed of carcinomatous and sarcomatoid elements. Sarcomatoid hepatocellular carcinoma (SHC), a type of sarcomatoid carcinoma composed of malignant spindle cells, was included in the World Health Organization's classification of liver tumors in 2010 (1). Previous reports of SHC have consisted mostly of single case reports, and have referred to SHC by various names, including spindle cell carcinoma (2), sarcomatoid carcinoma (3), pseudosarcoma (4), and carcinosarcoma (5).

Histologically, the most significant pathological feature of SHC is the presence of transitional zones between epithelial and mesenchymal cells; however, these zones are not always observed (6). Diagnosis of SHC is confirmed by spindle cell composition in hepatocellular carcinoma (HCC) $(1,7)$, and no valuable tumor markers associated with SHC have been reported thus far.

In clinical settings, SHC has been found in $3.99 .4 \%$ of HCC autopsies (8) and approximately $1.8 \%$ of surgically resected HCC specimens $(2,9)$. The clinical manifestations of SHC are similar to those of conventional HCC (2,3,9-12), but the scant data available suggest that the prognosis is significantly poorer $(9,12,13)$. Apart from radical resection, no effective treatment or prognosis prediction model for SHC has been established to date.

In the present study, we assessed the clinical features of SHC and examined some pathological markers of primary liver cancers to gain a better understanding of this rare tumor. We present the following article in accordance with the TRIPOD reporting checklist (available at https://atm. amegroups.com/article/view/10.21037/atm-21-4322/rc).

\section{Methods}

\section{Patients}

The study was conducted in accordance with the Declaration of Helsinki (as revised in 2013). The study was approved by the Institutional Ethics Committee of Zhongshan Hospital, Fudan University (No. B2021774) and individual consent for this retrospective analysis was waived. From April 2007 to November 2016, 12,012 consecutive patients who underwent surgical resection were diagnosed with HCC after pathological examination in the Department of Liver Surgery, Zhongshan Hospital, Fudan University. A total of 69 patients with SHC were subsequently identified from this initial patient population. The cumulative incidence of SHC in patients with surgically resected $\mathrm{HCC}$ was $0.57 \%$. We excluded 4 patients with a previous history of primary lung cancer or rectal cancer, and 2 patients were lost to follow-up. The medical records of the 63 remaining patients were retrospectively reviewed. To establish a nomogram that would serve as a quantitative tool to predict survival of patients with SHC, the patients were divided into a training set $(n=47)$ and an independent internal validation set $(n=16)$ at a ratio of $3: 1$. All patients were followed-up until June 2019. A flowchart of the patient selection process is shown in Figure 1 .

\section{Treatment}

All patients underwent routine examinations prior to the surgical resection, including liver function tests, tumor marker tests, hepatitis serology, chest radiography, and abdominal computed tomography or magnetic resonance imaging scanning. The treatment strategy was similar to that of conventional HCC and involved a hepatectomy procedure that has been previously described in detail (14). After surgery, any patient who met the antiviral therapy criteria of the Asian Pacific Association for the Study of the Liver (15) received either lamivudine (100 mg) or entecavir $(0.5 \mathrm{mg})$ daily.

\section{Endpoints}

The primary endpoint of the present study was overall survival (OS). The secondary endpoint was recurrencefree survival (RFS). OS was defined as the time from the date of surgery until the date of the last follow-up or death, regardless of the cause of death. RFS was defined as the time from the date of surgery to the date of the first documented disease recurrence by independent radiological or pathological assessment, or death by any cause, whichever occurred first (16).

\section{Definition and diagnosis}

The tumors were identified by imaging studies, and diagnoses for all patients were based on the pathological and immunohistochemical (IHC) staining results. SHC was diagnosed as conventional HCC with spindle cell foci (1), with the spindle cell component staining positive for keratin $(1,7)$. Microvascular invasion was determined according to methods described in previous studies (17). 


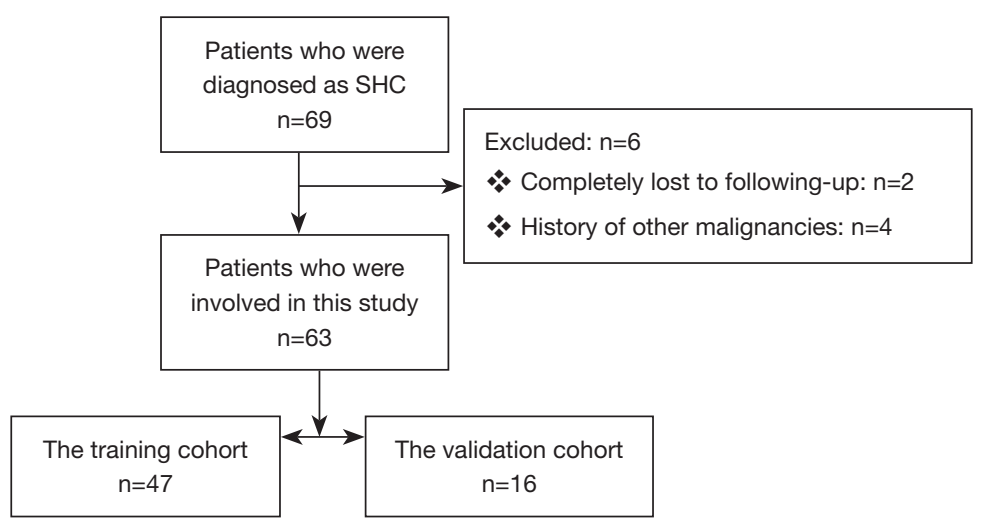

Figure 1 Patient selection flowchart. SHC, sarcomatoid hepatocellular carcinoma.

\section{IHC staining}

Immunostaining for biomarkers was performed on whole paraffin-embedded liver sections. The primary antibodies used are listed in Table S1. All immunostaining results were independently reviewed by 2 pathologists. If greater than $10 \%$ of the tumor cells showed cytoplasmic staining for heat-shock protein 70 (HSP70), glutamine synthetase (GS), or glypican-3 (GPC-3), the results were considered positive. Only those cases showing positive cytoplasmic staining in greater than $5 \%$ of tumor cells were considered positive for hepatocyte paraffin 1 (HepPar1), alphafetoprotein (AFP), cytokeratin 7 (CK7), or cytokeratin 19 (CK19). Positive immunostaining for Ki-67 was defined as the presence of nuclear staining. The expression level of tyrosine-protein kinase Met (c-Met) was evaluated by an $\mathrm{H}$-score assessment of its staining intensity (03) and the percentage of positively stained cells (0-100\%) (18). Each intensity level was multiplied by the percentage of positively stained cells, and all values were added to obtain the final IHC score (0 to 300). IHC scores over 150 were considered positive for c-Met staining. The final score was calculated from the assessment scores of the membrane and cytoplasmic expression levels (Figure 2A). The staining intensity of programmed death ligand-1 (PD-L1) expression was assessed in neoplastic cells (Figure 2B). The percentage of cells displaying unequivocal membranous staining was recorded, with tumors containing more than $1 \%$ of stained cells classified as positive (19).

\section{Data collection and follow-up}

The medical records of all patients were retrospectively reviewed. Patients were followed-up in our clinic every 23 months during the first year and once every 3-4 months thereafter. The results of the liver function tests, tumor marker analyses, and haematological parameters were examined. Computed tomography of the chest, abdomen, and pelvis was performed once every 6 months. If tumor recurrence to the liver was suspected, computed tomography, magnetic resonance imaging, or biopsy of the lesions was performed to exclude metastasis.

\section{Statistical analysis}

The baseline patient characteristics are reported as the median (range) or percentage, as appropriate. The best cut-off values for continuous variables were obtained using X-tile, version 3.6.1 (Yale University, New Haven, CT, USA). The Student's $t$-test was employed to compare continuous variables. OS and RFS were examined using the Kaplan-Meier method with log-rank tests. A nomogram was formulated based on the results of the multivariate analysis using the rms package in $\mathrm{R}$, version 3.6.3 (The $\mathrm{R}$ Foundation for Statistical Computing, Vienna, Austria). The final selection of the model was performed using a backward stepdown selection process with the Akaike information criterion. Bootstrap resampling of 1,000 samples was used for these activities. The larger the concordance index (C-index), the more accurate the prognostic prediction. During the validation of our nomogram, the total points for each patient in the validation set were calculated according to the established nomogram, and then a Cox regression of this set was performed using the total points as a factor. A P value less than 0.05 was considered statistically significant. 
A
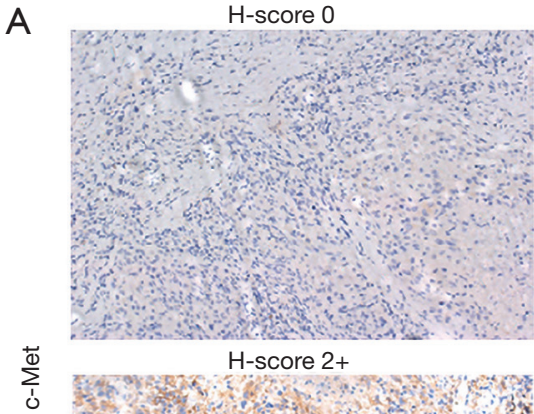

$\frac{5}{3}$

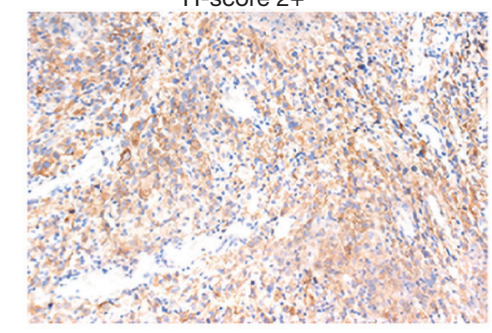

B
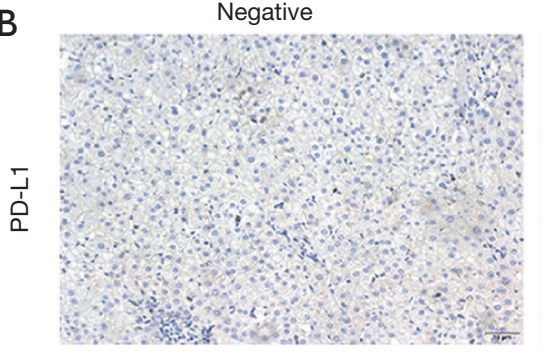

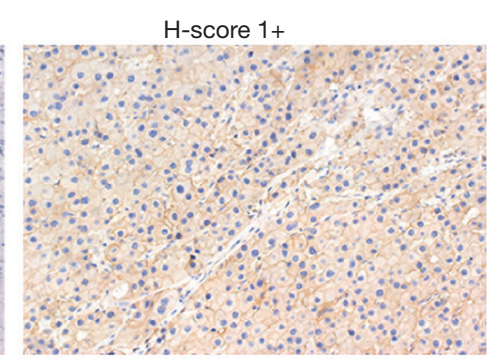

$\mathrm{H}$-score $3+$

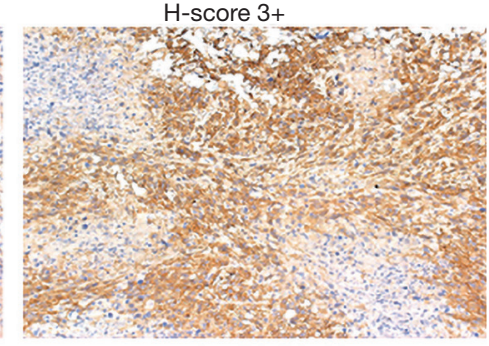

Positive

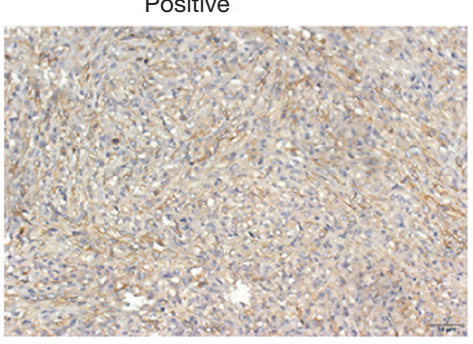

Figure 2 Typical pathology pictures. Consecutive sections were used for immunohistochemical study on expression of c-Met (A) and PD-L1 (B). Positive cells were stained brown. Magnification: ×200. PD-L1, programmed death ligand-1.

\section{Results}

\section{Clinical characteristics}

Clinical characteristics of patients were summarized in Table 1 . The median age was 59 years (range, $35-78$ years), and the sex ratio (male:female) was 2.3:1.0 (44:19). Most patients $(68.3 \%, 43 / 63)$ were asymptomatic and had their tumors discovered during a routine medical examination. The hepatitis B surface antigen was detected in $71.4 \%(45 / 63)$ SHC with SHC; the corresponding statistic in our hospital was $76.8 \%$ (721/939) (14). The percentages of patients who were positive for serum AFP (>20 ng/mL), serum carcinoembryonic antigen $(>5 \mathrm{ng} / \mathrm{mL})$, and serum carbohydrate antigen $19-9$ (>37 ng/mL) were $36.5 \%$ (23/63), $7.9 \%$ (5/63), and $19.0 \%$ (12/63), respectively. In contrast, AFP positivity has been found in $67.9 \%(679 / 1,000)$ of patients with conventional HCC (14). Extrahepatic spread was found in 11 patients (6 to lymph nodes, 2 to adrenal glands, 2 to the diaphragm, and 1 to the greater omentum). According to the Child-
Pugh classification, $81.0 \%(51 / 63)$ of patients could be categorized as class A and $19.0 \%$ (12/63) of patients could be categorized as class $\mathrm{B}$.

\section{Histological and pathological findings}

Some IHC markers relating to the diagnosis or prognosis of primary liver cancers were examined. IHC analyses showed that HepPar1 was positive in only $38.1 \%$ of patients (24/63) and AFP was positive in $11.1 \%$ of patients (7/63). GPC3, HSP70, and GS have been recommended as the most valuable diagnostic combination for HCC detection (20). GPC3, HSP70, and GS were positive in $41.3 \%(26 / 63)$, $82.5 \%(52 / 63)$, and $77.8 \%$ (49/63) of patients, respectively. The presence of at least two immunoreactive markers, which was used in early HCC diagnosis, was identified in $79.4 \%(50 / 63)$ of patients. Notably, the positivity of this panel indicated a better prognosis of SHC [hazard ratio $(\mathrm{HR})=0.31, \mathrm{P}<0.05]$. The expression of CK7 and CK19 was classified as positive in $55.6 \%(35 / 63)$ and $57.1 \%$ 
Table 1 Clinical characteristics of patients

\begin{tabular}{|c|c|c|c|c|}
\hline Variables & All patients $(n=63)$ & Training cohort $(n=47)$ & Validation cohort $(n=16)$ & $\mathrm{P}$ \\
\hline Sex, male/female & $19 / 44$ & $14 / 33$ & $5 / 11$ & 0.91 \\
\hline Tumor number, single/multiple & $16 / 47$ & $13 / 34$ & $3 / 13$ & 0.48 \\
\hline Tumor size, cm, median [range] & $6.0[1.5-18.0]$ & $6.0[1.5-12.0]$ & $5.5[2.2-18.0]$ & 0.07 \\
\hline AFP, $<20 / \geq 20 \mathrm{ng} / \mathrm{mL}$ & $40 / 23$ & $30 / 17$ & $10 / 6$ & 0.92 \\
\hline Total bilirubin, $<20.4 / \geq 20.4 \mu \mathrm{mol} / \mathrm{L}$ & $56 / 7$ & $41 / 6$ & $15 / 1$ & 0.80 \\
\hline Albumin, $<35 / \geq 35 \mathrm{~g} / \mathrm{L}$ & $21 / 42$ & $15 / 32$ & $6 / 10$ & 0.68 \\
\hline INR, $<1.2 / \geq 1.2$ & $56 / 7$ & $42 / 5$ & $14 / 2$ & 0.80 \\
\hline HbsAg, negative/positive & $18 / 45$ & $11 / 36$ & $7 / 9$ & 0.22 \\
\hline MVI, negative/positive & $33 / 30$ & $22 / 25$ & $11 / 5$ & 0.13 \\
\hline Cirrhosis, negative/positive & $29 / 34$ & $19 / 28$ & $10 / 6$ & 0.13 \\
\hline Tumor capsule, complete/incomplete & $19 / 44$ & $13 / 34$ & $6 / 10$ & 0.67 \\
\hline HSP70, negative/positive & $52 / 11$ & $40 / 7$ & $12 / 4$ & 0.36 \\
\hline GS, negative/positive & $49 / 14$ & $36 / 11$ & $13 / 3$ & 0.70 \\
\hline GPC3, negative/positive & $26 / 37$ & $19 / 28$ & $7 / 9$ & 0.82 \\
\hline $\begin{array}{l}\text { Two positive markers out of HSP70, GP3, GS), } \\
\text { yes/no }\end{array}$ & $50 / 13$ & $38 / 9$ & $12 / 4$ & 0.62 \\
\hline
\end{tabular}

AFP, alpha-fetoprotein; INR, international normalized rate; CEA, carcino-embryonic antigen; CA199, carbohydrate antigen 199; HBsAg, hepatitis B surface antigen; MVI, microvascular invasion; AJCC, American Joint Committee on Cancer; BCLC, Barcelona Clinic Liver Cancer; HSP70, heat-shock protein 70; GS, glutamine synthetase; GPC3, glypican-3.

(36/63) of patients, respectively. In addition, 34.9\% (22/63) patients were CK7 and CK19 double-positive. C-Met is a therapeutically relevant target in HCC, with important roles in proliferation, motility, and invasion. Overall, 27.0\% (17/63) of patients were positive for c-Met. High c-Met expression levels were associated with larger tumor size $(\mathrm{P}=0.05)$. $\mathrm{PD}-\mathrm{L} 1$, an important immune checkpoint, was positive in $19.0 \%$ (12/63) of patients. Approximately $90.5 \%$ (57/63) of patients had a high level of Ki-67.

\section{Outcomes and factors associated with patient survival}

With a follow-up period lasting a median of 37.3 months (range, 1.4-55.6 months), the median OS and RFS in the 63 study patients were 23.2 and 8.4 months, respectively. The 1-, 2-, and 3-year OS rates were 59.0\%, 45.4\%, and 34.4\%, respectively. The 1-, 2-, and 3-year RFS rates were $36.3 \%$, $26.1 \%$, and $22.4 \%$, respectively. Thirty-day morbidity was negligible. 
Table 2 Univariate and multivariate analysis for overall survival in all patients

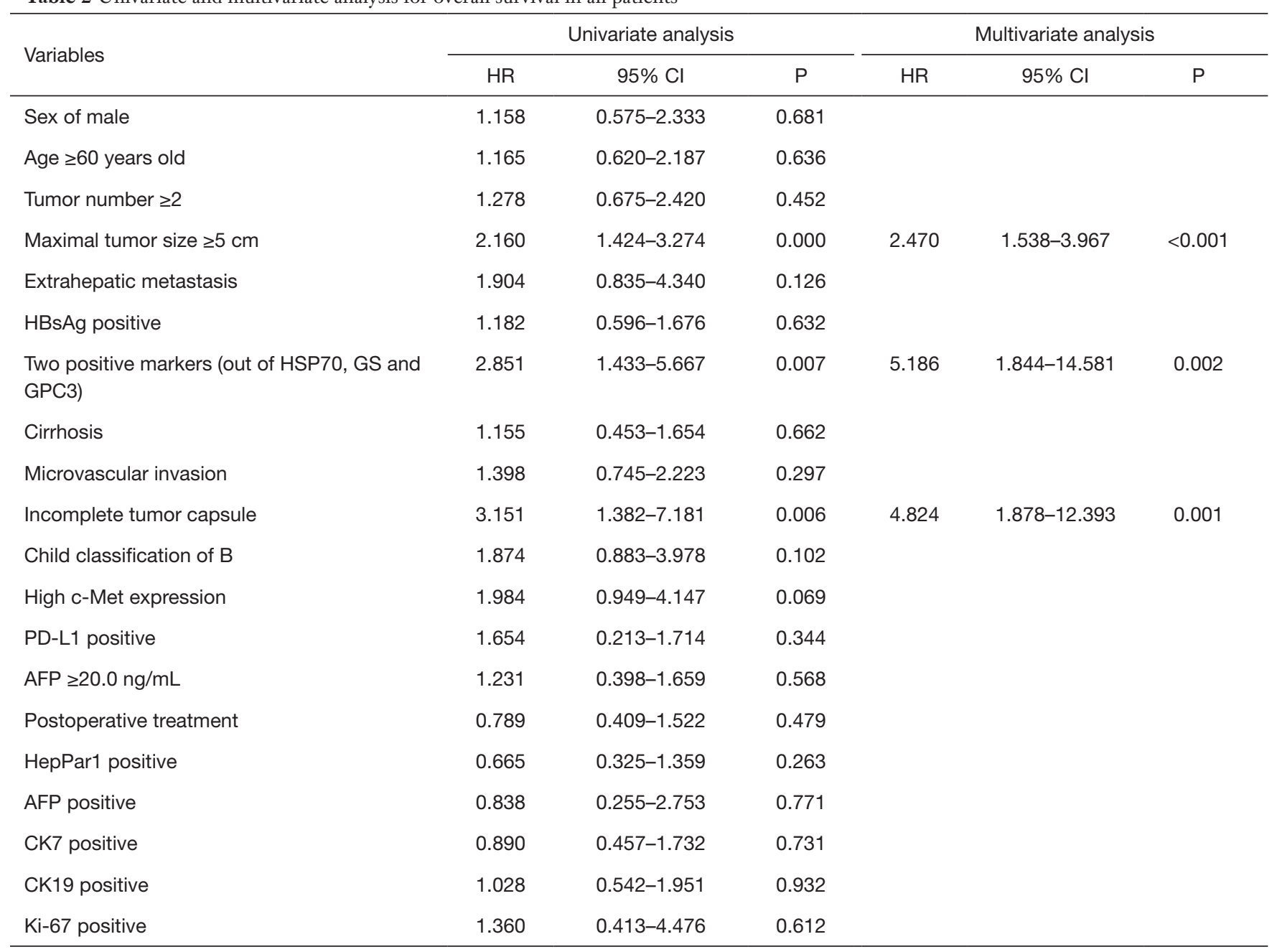

HR, hazard ratio; Cl, confidence interval; HBsAg, Hepatitis B surface antigen; HSP70, heat-shock protein 70; GS, glutamine synthetase; GPC3, glypican-3; PD-L1, programmed death ligand-1; AFP, alpha-fetoprotein; HepPar1, hepatocyte paraffin 1; CK, cytokeratin.

The results of the univariate and multivariate analyses of OS and RFS are listed in Tables 2,3. In the OS univariate analysis of all patients, maximal tumor size, the presence of a tumor capsule, and the panel of GPC3, HSP70, and GS biomarkers were all associated with survival (all $\mathrm{P}<0.05$ ). In the RFS univariate analysis, 5 risk factors, including the presence of a tumor capsule, maximal tumor size, ChildPugh classification, c-Met expression level, and the GPC3, HSP70, and GS biomarkers were all associated with RFS (all $\mathrm{P}<0.05)$. Postoperative treatments, including transarterial chemoembolization (TACE) and chemotherapy, did not confer any survival advantage (all $\mathrm{P}>0.05$ ). In the adjusted Cox proportional hazards model, an incomplete tumor capsule, a larger maximal tumor size, and the presence of 2 positive markers (out of GPC3, HSP70, and GS) were all associated with poorer OS and RFS (Tables 2,3).

\section{Development and validation of the prognostic nomogram}

The clinical characteristics of patients with SHC in the training and validation sets are summarized in Table 1. In the training set, the follow-up period lasted a median of 38.4 months (range, 1.4-55.6 months). The 1-, 2-, and 3-year OS rates were $56.5 \%, 45.1 \%$, and $30.5 \%$, respectively.

OS univariate analysis of patients in the training set revealed that maximal tumor size, the presence of a tumor capsule, Child-Pugh classification, and a combination of the markers HSP70, GS, and GPC3 were all associated 
Table 3 Univariate and multivariate analysis for recurrence-free survival in all patients

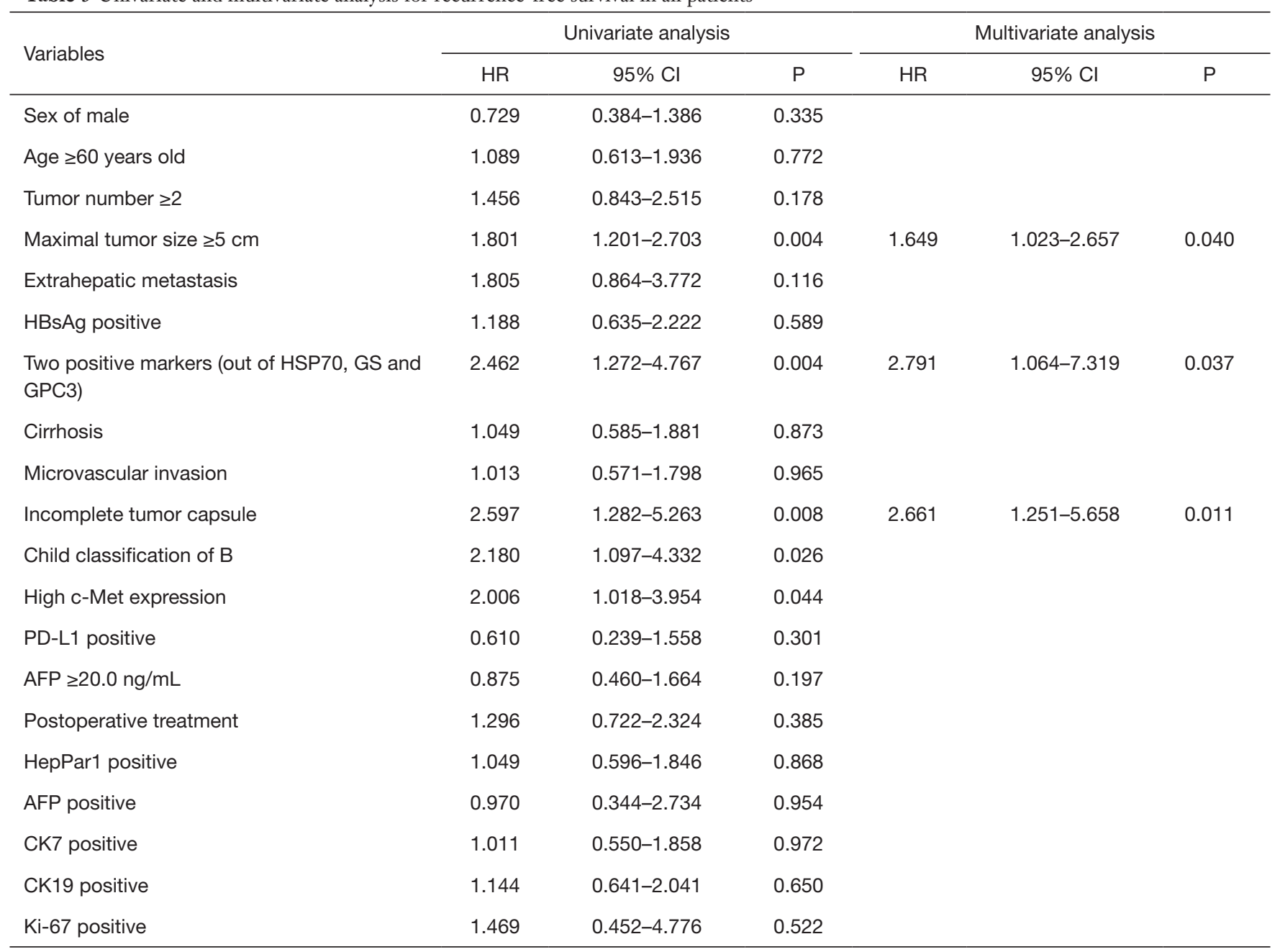

HR, hazard ratio; Cl, confidence interval; HBsAg, Hepatitis B surface antigen; HSP70, heat-shock protein 70; GS, glutamine synthetase; GPC3, glypican-3; PD-L1, programmed death ligand-1; AFP, alpha-fetoprotein; HepPar1, hepatocyte paraffin 1; CK, cytokeratin.

with an increased mortality rate (all $\mathrm{P}<0.05)$. The other characteristics listed in Table 4 were not considered significant predictors of OS. Factors with a $\mathrm{P}$ value less than 0.10 were included in the multivariate analysis, and maximal tumor size, the presence of a tumor capsule, and 2 positive immunoreactive markers (out of GPC3, HSP70, and GS) were independent risk factors for OS (Table 4).

A prognostic nomogram that integrated all significant independent factors for 1 - and 2-year OS in the training set is shown in Figure 3. The C-index for OS prediction was 0.758 (95\% CI: $0.672-0.843)$. A calibration plot for the probability of survival at 1 and 2 years after surgery showed an optimal agreement between the prediction by the nomogram and actual observations (Figure 4).
In the validation set, the median OS was 26.9 months (range, 1.4-55.3 months), and the postoperative 1-, 2-, and 3 -year OS rates were $75.0 \%, 58.3 \%$, and $33.3 \%$, respectively. The $\mathrm{C}$-index of the nomogram for predicting OS was 0.832 (95\% CI: 0.712-0.952). A calibration curve showed good agreement between the prediction of survival by the nomogram and observations of the probability of 1-year survival (Figure 5).

\section{Comparison of predictive accuracy between the nomogram and conventional staging systems}

Despite similar clinical symptoms, the clinical and pathological characteristics of SHC differ from those of 
Table 4 Univariate and multivariate analysis for overall survival in the training cohort

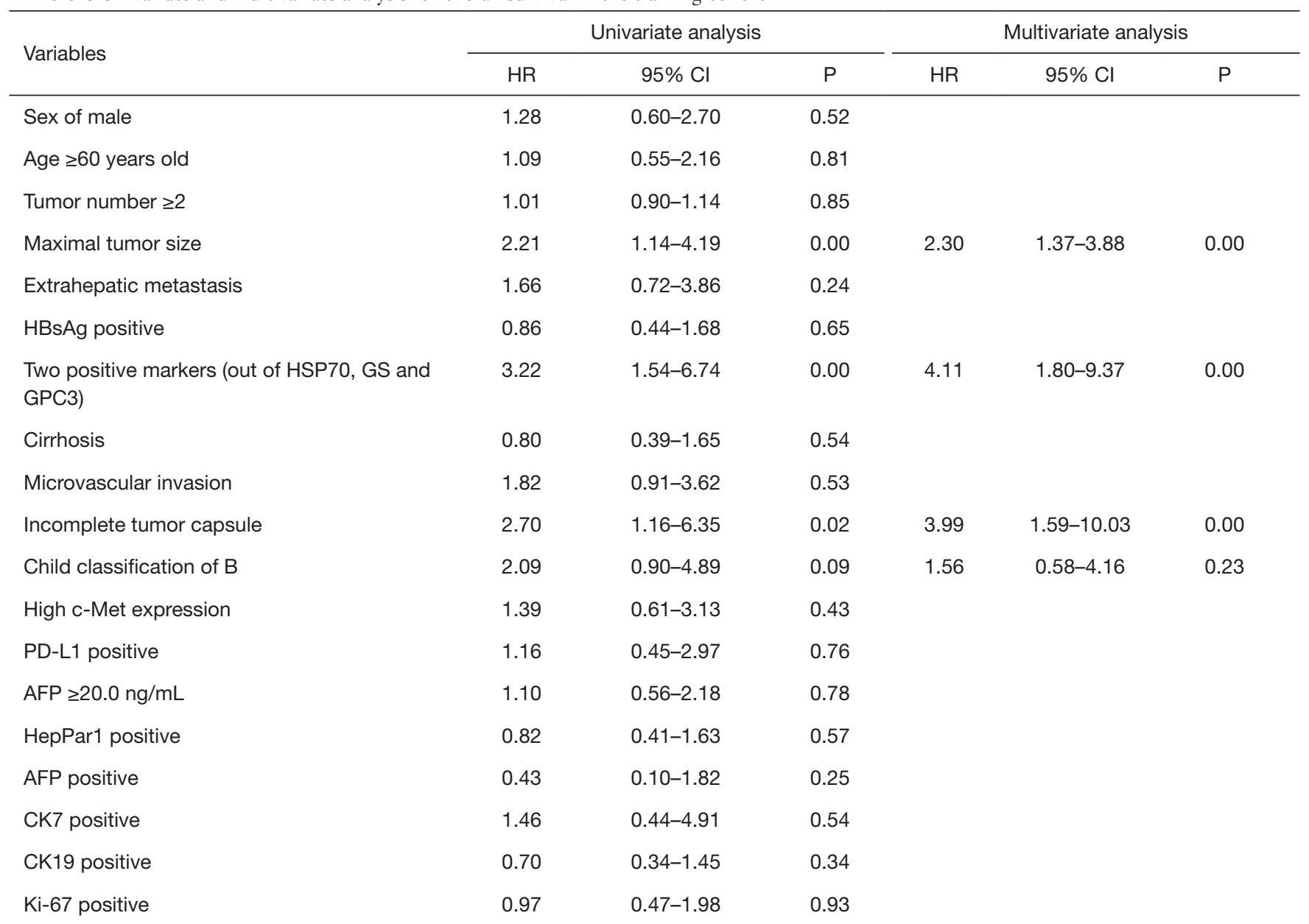

HR, hazard ratio; Cl, confidence interval; HBsAg, Hepatitis B surface antigen; HSP70, heat-shock protein 70; GS, glutamine synthetase; GPC3, glypican-3; PD-L1, programmed death ligand-1; AFP, alpha-fetoprotein; HepPar1, hepatocyte paraffin 1; CK, cytokeratin.

HCC (13). Therefore, conventional staging systems may not be appropriate for SHC. The C-index of our nomogram was 0.758 (95\% CI: 0.672-0.843), which was higher than that of other staging systems, including the BarcelonaClinical Liver Cancer (BCLC) staging system (21), and the American Joint Committee on Cancer (AJCC), eighth edition (22). The $\mathrm{C}$-indices of these staging systems were 0.591 (BCLC) and 0.587 (AJCC) in all patients. The $\mathrm{C}$-index of our nomogram was significantly higher than that of conventional staging systems for HCC (all $\mathrm{P}<0.05$ ). The addition of the panel of markers (GPC3, HSP70, and GS) improved the prognostic accuracy of the nomogram model.

\section{Discussion}

The present study, which is the largest to date investigating pathological features of SHC, included 63 who were diagnosed with SHC between 2007 and 2016. The clinical symptoms of SHC in our study were similar to those of conventional HCC, as most cases (43/63) were asymptomatic and were found during routine examinations. However, the serum tumor markers and immunohistochemical markers differ significantly between SHC and conventional HCC. In conventional HCC, abnormal AFP ( $>20 \mathrm{ng} / \mathrm{mL}$ ) levels were found in more than $70 \%$ of patients (23). In our study, only $36.5 \%$ of patients with SHC showed abnormal AFP levels. Similarly, in conventional HCC, HepPar1 and AFP both have a high positive rate, with a rate of $100 \%(50 / 50)$ for HepPar1 (20) and $56.8 \%$ (21/37) for AFP (21). In our study, the positive rates of HepPar1 and AFP were both very low. The combination of GPC3, HSP70, and GS has been used in 


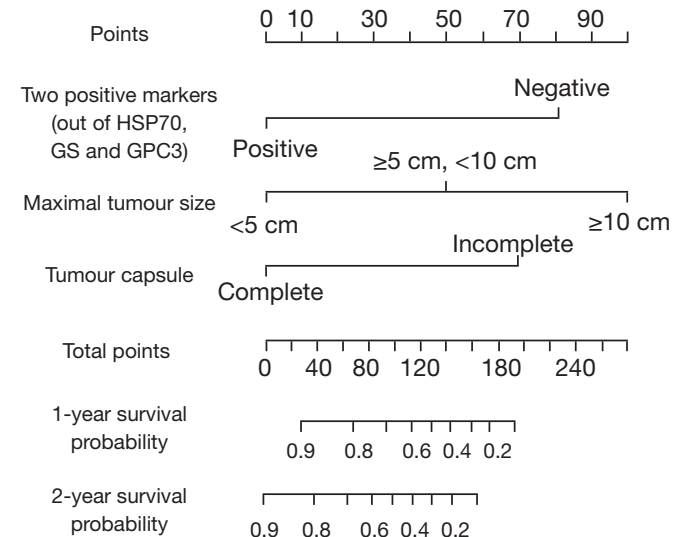

Figure 3 Sarcomatoid hepatocellular carcinoma survival nomogram. To use the nomogram, calculate the points identified on the points scale for each variable and figure up all points. On the bottom scales, the nomogram can provide 1- and 2-year survival probability for an individual patient. HSP70, heat-shock protein 70; GS, glutamine synthetase; GPC3, glypican-3.

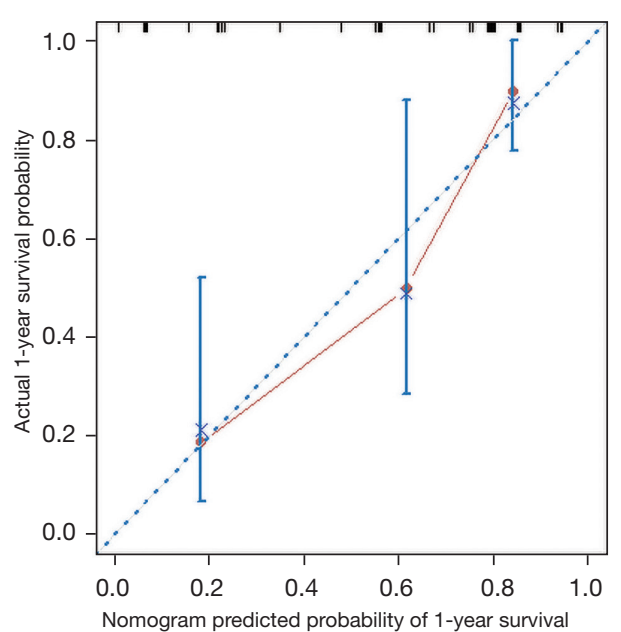

Figure 4 The calibration curve for predicting 1-year survival probability in patients with sarcomatoid hepatocellular carcinoma by Bootstrap validation. The $\mathrm{x}$-axis represents the nomogrampredicted survival, and the actual survival is plotted on the $y$-axis.

the detection of early HCC with an overall accuracy of $78.4 \%$ ( 2 markers positive) with $100 \%$ specificity (20). In our study of SHC, the positive rate of this panel was $79.4 \%$ (50/63) in SHC, while the negativity of this panel indicated a poorer outcome. Positive staining for HSP70 or GPC3 has been reported to be an indicator of poor survival in HCC $(24,25)$, but overall mortality is lower in GS-positive

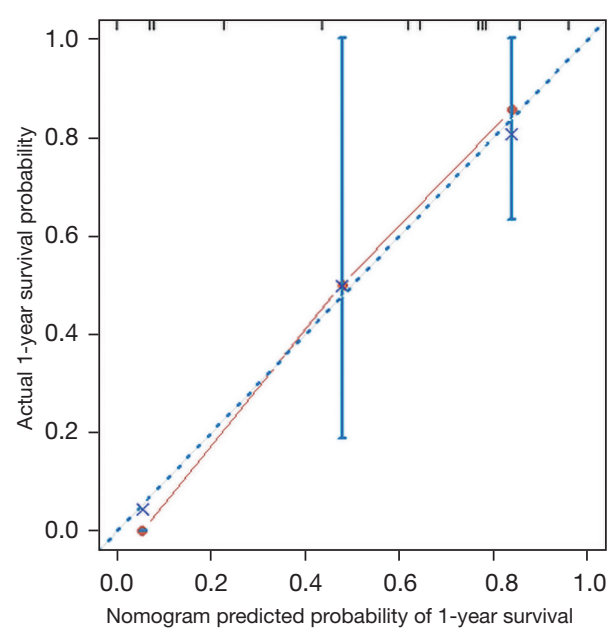

Figure 5 The calibration curve for predicting 1-year survival probability in the validation cohort. The $\mathrm{x}$-axis represents the nomogram-predicted survival, and the actual survival was plotted on the y-axis.

patients (26). In our study of SHC, positive staining of any 1 of these 3 markers was unrelated to survival time. These results suggest that SHC displays different pathological features to conventional HCC with poorly differentiated histology.

Our study found that the prognosis of SHC is significantly poorer than that of conventional HCC, which is consistent with findings from previous studies $(3,10,11,13)$. In our hospital, the 3 -year survival rate of conventional HCC was found to be $61.0 \%$ in our hospital (14), whereas it was only $28.2 \%$ for patients with SHC in the present study. Patients had a high rate of early tumor recurrence: more than half of the patients (37/63) had tumor recurrence or died within 1 year of surgical resection. The poor prognosis suggests that the efficacies of hepatic resection and postoperative treatments are very limited. Effective postoperative adjuvant treatments are essential to improving the outcomes of patients with SHC. However, no specific treatments for SHC are currently available. A recent study showed that patients with SHC who received adjuvant treatments after surgery had better outcomes than those who did not receive any postoperative treatment (11), but no details regarding treatment strategies were provided. In the present study, postoperative treatment, including TACE and chemotherapy, did not provide better patient outcomes $(\mathrm{P}>0.05)$.

Over the past few decades, research on cancer 
therapeutics has mainly focused on targeted therapies and immunotherapies. c-Met was identified as a prognostic factor for HCC in 2015 (27), and it is still an active target for research in HCC. Previous studies have reported that selective c-Met inhibitors have antitumor activity in HCC and a favourable safety profile $(28,29)$. More importantly, the activation of mesenchymal-epithelial transition (MET) in HCC indicates the resistance to sorafenib but not to cabozantinib, which has significant antitumor effects in HCC (30). In the present study, high c-Met expression levels were associated with larger tumor size $(\mathrm{P}=0.05)$, which suggests that c-Met activation results in tumor proliferation in SHC (31). High levels of c-Met indicated poorer RFS in the univariate analysis $(\mathrm{P}<0.05)$. Immune checkpoint inhibitors that target programmed cell death protein 1 (PD-1) have ushered in a new era of cancer therapies. Several studies have shown that high expression levels of PD-L1 are significantly correlated with unfavourable survival in patients with HCC $(19,32,33)$. We found that while approximately $19.0 \%$ (12/63) of patients were positive for PD-L1, this finding was not associated with OS or RFS in these patients (all $\mathrm{P}>0.05$ ).

The prognosis for patients with SHC differs from that of conventional HCC (13). Conventional staging systems may not be applicable to SHC. In this study, the C-indices of all staging systems for patients with SHC were low (all $\mathrm{C}$-indices $<0.70$ ). Thus, we established a new prognostic model for SHC. Three variables were identified as critical for the prognostic score: maximal tumor size, the presence of a tumor capsule, and the panel of IHC markers (GPC3, HSP70, and GS). The presence of a tumor capsule has generally been ignored in prior predictions of survival but was tightly related to OS in SHC. The current study's nomogram model offers superior prognostic performance for patients with SHC and may provide valuable information for outcome prediction in such patients.

Two limitations should be noted. The case numbers of SHC were very limited, and the referral bias could not be completely avoided.

In conclusion, SHC can be characterized as a rare subtype of HCC with different pathological features and extremely poor prognosis. A panel of markers, HSP 70, GS, and GPC3, can serve as an independent prognostic factor for SHC.

\section{Acknowledgments}

Funding: This work was supported by the National
Science and Technology Major Project (grant No. 2018ZX10723204-004 to Z Wang) and the Shanghai Natural Science Foundation (grant No. 20ZR1411400 to Z Wang).

\section{Footnote}

Reporting Checklist: The authors have completed the TRIPOD reporting checklist. Available at https://atm. amegroups.com/article/view/10.21037/atm-21-4322/rc

Data Sharing Statement: https://atm.amegroups.com/article/ view/10.21037/atm-21-4322/dss

Peer Review File: Available at https://atm.amegroups.com/ article/view/10.21037/atm-21-4322/prf

Conflicts of Interest: All authors have completed the ICMJE uniform disclosure form (available at https://atm. amegroups.com/article/view/10.21037/atm-21-4322/coif). The authors have no conflicts of interest to declare.

Ethical Statement: The authors are accountable for all aspects of the work in ensuring that questions related to the accuracy or integrity of any part of the work are appropriately investigated and resolved. The study was conducted in accordance with the Declaration of Helsinki (as revised in 2013). The study was approved by the Institutional Ethics Committee of Zhongshan Hospital, Fudan University (No. B2021-774) and individual consent for this retrospective analysis was waived.

Open Access Statement: This is an Open Access article distributed in accordance with the Creative Commons Attribution-NonCommercial-NoDerivs 4.0 International License (CC BY-NC-ND 4.0), which permits the noncommercial replication and distribution of the article with the strict proviso that no changes or edits are made and the original work is properly cited (including links to both the formal publication through the relevant DOI and the license). See: https://creativecommons.org/licenses/by-nc-nd/4.0/.

\section{References}

1. Bosman FT, Carneiro F, Hruban RH, et al. WHO classification of tumours of the digestive system. 4th edition. Geneva, Switzerland: World Health Organization, 2010. 
2. Maeda T, Adachi E, Kajiyama K, et al. Spindle cell hepatocellular carcinoma. A clinicopathologic and immunohistochemical analysis of 15 cases. Cancer 1996;77:51-7.

3. Wang QB, Cui BK, Weng JM, et al. Clinicopathological characteristics and outcome of primary sarcomatoid carcinoma and carcinosarcoma of the liver. J Gastrointest Surg 2012;16:1715-26.

4. McCort JJ. Esophageal carcinosarcoma and pesudosarcoma. Radiology 1972;102:519-24.

5. Lao XM, Chen DY, Zhang YQ, et al. Primary carcinosarcoma of the liver: clinicopathologic features of 5 cases and a review of the literature. Am J Surg Pathol 2007;31:817-26.

6. Sung CO, Choi H, Lee KW, et al. Sarcomatoid carcinoma represents a complete phenotype with various pathways of epithelial mesenchymal transition. J Clin Pathol 2013;66:601-6.

7. Torbenson MS. Morphologic Subtypes of Hepatocellular Carcinoma. Gastroenterol Clin North Am 2017;46:365-91.

8. Kojiro M, Sugihara S, Kakizoe S, et al. Hepatocellular carcinoma with sarcomatous change: a special reference to the relationship with anticancer therapy. Cancer Chemother Pharmacol 1989;23 Suppl:S4-8.

9. Nishi H, Taguchi K, Asayama Y, et al. Sarcomatous hepatocellular carcinoma: a special reference to ordinary hepatocellular carcinoma. J Gastroenterol Hepatol 2003;18:415-23.

10. Chin S, Kim Z. Sarcomatoid combined hepatocellularcholangiocarcinoma: a case report and review of literature. Int J Clin Exp Pathol 2014;7:8290-4.

11. Kan A, Guo RP. The prognosis of subsequent surgical treatment in patients with sarcomatoid carcinoma in the liver: A retrospective study. Int J Surg 2018;55:145-51.

12. Koo HR, Park MS, Kim MJ, et al. Radiological and clinical features of sarcomatoid hepatocellular carcinoma in 11 cases. J Comput Assist Tomogr 2008;32:745-9.

13. Liao SH, Su TH, Jeng YM, et al. Clinical Manifestations and Outcomes of Patients with Sarcomatoid Hepatocellular Carcinoma. Hepatology 2019;69:209-21.

14. Zhou XD, Tang ZY, Yang BH, et al. Experience of 1000 patients who underwent hepatectomy for small hepatocellular carcinoma. Cancer 2001;91:1479-86.

15. Sarin SK, Kumar M, Lau GK, et al. Asian-Pacific clinical practice guidelines on the management of hepatitis B: a 2015 update. Hepatol Int 2016;10:1-98.

16. Cucchetti A, Piscaglia F, Cescon M, et al. Costeffectiveness of hepatic resection versus percutaneous radiofrequency ablation for early hepatocellular carcinoma. J Hepatol 2013;59:300-7.

17. Rodríguez-Perálvarez M, Luong TV, Andreana L, et al. A systematic review of microvascular invasion in hepatocellular carcinoma: diagnostic and prognostic variability. Ann Surg Oncol 2013;20:325-39.

18. Shao YY, Hsiao CH, Lee R, et al. Tumor c-Met expression and prognosis of advanced hepatocellular carcinoma patients treated with sorafenib. J Clin Oncol 2015;33:abstr 317.

19. Calderaro J, Rousseau B, Amaddeo G, et al. Programmed death ligand 1 expression in hepatocellular carcinoma: Relationship With clinical and pathological features. Hepatology 2016;64:2038-46.

20. Di Tommaso L, Destro A, Seok JY, et al. The application of markers (HSP70 GPC3 and GS) in liver biopsies is useful for detection of hepatocellular carcinoma. J Hepatol 2009;50:746-54.

21. Cillo U, Vitale A, Grigoletto F, et al. Prospective validation of the Barcelona Clinic Liver Cancer staging system. J Hepatol 2006;44:723-31.

22. Chun YS, Pawlik TM, Vauthey JN. 8th Edition of the AJCC Cancer Staging Manual: Pancreas and Hepatobiliary Cancers. Ann Surg Oncol 2018;25:845-7.

23. Zhou J, Sun HC, Wang Z, et al. Guidelines for Diagnosis and Treatment of Primary Liver Cancer in China (2017 Edition). Liver Cancer 2018;7:235-60.

24. Shin E, Ryu HS, Kim SH, et al. The clinicopathological significance of heat shock protein 70 and glutamine synthetase expression in hepatocellular carcinoma. J Hepatobiliary Pancreat Sci 2011;18:544-50.

25. Fu SJ, Qi CY, Xiao WK, et al. Glypican-3 is a potential prognostic biomarker for hepatocellular carcinoma after curative resection. Surgery 2013;154:536-44.

26. Dal Bello B, Rosa L, Campanini N, et al. Glutamine synthetase immunostaining correlates with pathologic features of hepatocellular carcinoma and better survival after radiofrequency thermal ablation. Clin Cancer Res 2010;16:2157-66.

27. Kang YK, Yau T, Park JW, et al. Randomized phase II study of axitinib versus placebo plus best supportive care in second-line treatment of advanced hepatocellular carcinoma. Ann Oncol 2015;26:2457-63.

28. Bouattour M, Raymond E, Qin S, et al. Recent developments of c-Met as a therapeutic target in hepatocellular carcinoma. Hepatology 2018;67:1132-49.

29. Zhou KQ, Liu WF, Yang LX, et al. Circulating osteopontin per tumor volume as a prognostic biomarker 
for resectable intrahepatic cholangiocarcinoma. Hepatobiliary Surg Nutr 2019;8:582-96.

30. Xiang Q, Chen W, Ren M, et al. Cabozantinib suppresses tumor growth and metastasis in hepatocellular carcinoma by a dual blockade of VEGFR2 and MET. Clin Cancer Res 2014;20:2959-70.

31. Drilon A, Laetsch TW, Kummar S, et al. Efficacy of Larotrectinib in TRK Fusion-Positive Cancers in Adults and Children. N Engl J Med 2018;378:731-9.

Cite this article as: Zhou C, Zhang X, Zhou K, Hou Y, Chen F, Zhang X, Ji Y, Qiu S, Fan J, Zhou J, Zhou Y, Wang Z. Long-term outcomes and prognosis for patients with sarcomatoid hepatocellular carcinoma. Ann Transl Med 2022;10(7):394. doi: 10.21037/atm-21-4322
32. Gao Q, Wang XY, Qiu SJ, et al. Overexpression of PDL1 significantly associates with tumor aggressiveness and postoperative recurrence in human hepatocellular carcinoma. Clin Cancer Res 2009;15:971-9.

33. Ma LJ, Feng FL, Dong LQ, et al. Clinical significance of PD-1/PD-Ls gene amplification and overexpression in patients with hepatocellular carcinoma. Theranostics 2018;8:5690-702. 
Supplementary

Table S1 The primary antibodies used in this study

\begin{tabular}{lllc}
\hline Antibody & Clonality & Supplier & Dilution \\
\hline PD-L1 & $28-8$ & Abcam & $1: 100$ \\
C-Met & D1C2 & Cell Signaling Technology & $1: 100$ \\
HepPar1 & OCH1E5 & DAKO & $1: 100$ \\
AFP & ZSA06 & DAKO & $1: 400$ \\
CK7 & OV-TL & DAKO & $1: 100$ \\
CK19 & RCK108 & DAKO & $1: 100$ \\
Hsp70 & W27 & Santa Cruz Biotechnology & $1: 100$ \\
GPC3 & G12 & Gene Tech & $1: 100$ \\
GS & Polyclonal & Abcam & $1: 2,000$ \\
Ki-67 & MIB-1 & DAKO & $1: 100$ \\
\hline
\end{tabular}

